EPJ Web of Conferences 19, 03001 (2012)

DOI: $10.1051 /$ epjconf/20121903001

(C) Owned by the authors, published by EDP Sciences, 2012

\title{
Are dSph galaxies Galactic building blocks?
}

\author{
G. Gilmore ${ }^{1,2, a}$ and H.M. Asiri² \\ ${ }^{1}$ Institute of Astronomy, Cambridge, UK \\ ${ }^{2}$ Astronomy Department, Faculty of Science, King Abdulaziz University, PO Box 80203, \\ Jeddah 21589, Saudi Arabia
}

\begin{abstract}
Dwarf spheroidal galaxies (dSph) are frequently assumed to represent surviving examples of a vast now destroyed population of small systems in which many of the stars now forming the Milky Way were formed. Ongoing accretion and considerable sub-structure in the outer Galactic halo is direct evidence that there is some role for stars formed in small galaxies in populating the (outer) galaxy. The evidence from stellar populations is however contradictory to this. dSph stellar populations are unlike any stars found in significant numbers in the Milky Way. The dSph are indeed small galaxies, formed over long times with low rates of star formation. Most of the stars in the Milky Way halo however seem to have formed quickly, at higher star formation rate, in gas mixed efficiently on kpc scales. The overwhelming majority of Milky Way stars, those in the Galactic thick disk and thin disk, seem to have nothing at all to do with dwarf galaxy origins.
\end{abstract}

\section{INTRODUCTION}

There is overwhelming and irrefutable evidence that large scale structure in the Universe is welldescribed by the $\Lambda$ CDM model. In this a (primordial) spectrum of perturbations with equal power on all scales generates density fluctuations which grow under self-gravity, with the dominant matter being both non-relativistic at early times, and weakly or un-coupled to the radiation which dominates the Universe at those early times. Thus the dominant matter cannot be baryonic, which couples efficiently to radiation through Thompson scattering. It is non-relativistic, does not couple to, or emit, radiation, and dominates the local gravitational field. Hence Cold Dark Matter, CDM. This model describes the Universe well on large scales, in fact on all scales large enough that the growth of density perturbations remains linear, so that local dark matter particle physics and baryonic physical processes remain unimportant. The baryonic physics of gas cooling becomes very important at scales of order $10^{6} M_{\odot}$. There must also be some general small-scale limit, when the physical properties of whatever types of particles make up the CDM become important, and dominate the scale-free initial perturbations. Finding that scale will provide our first information on the types of matter which make up CDM, and so is of very considerable interest.

A standard approach to discovering that scale is to examine when an assumed absence of scale violates observation. That is, one extrapolates the model to smaller and smaller scales until observations indicate discordance. This is the particular interest of dwarf galaxies, which form the smallest systems in which dark matter has been detected. Are they consistent with extrapolated gravitational CDM and baryons, with no evidence of additional physics? The simplest, and most extreme, CDM extrapolation posits structure in gravitationally bound CDM potential wells on all scales, with the number of such systems increasing as mass ${ }^{-2}$. This is divergent, so must cut off somewhere. But at what scale does

\footnotetext{
ae-mail: gil@ast.cam.ac.uk
}

This is an Open Access article distributed under the terms of the Creative Commons Attribution-Noncommercial License 3.0, which permits unrestricted use, distribution, and reproduction in any noncommercial medium, provided the original work is properly cited. 


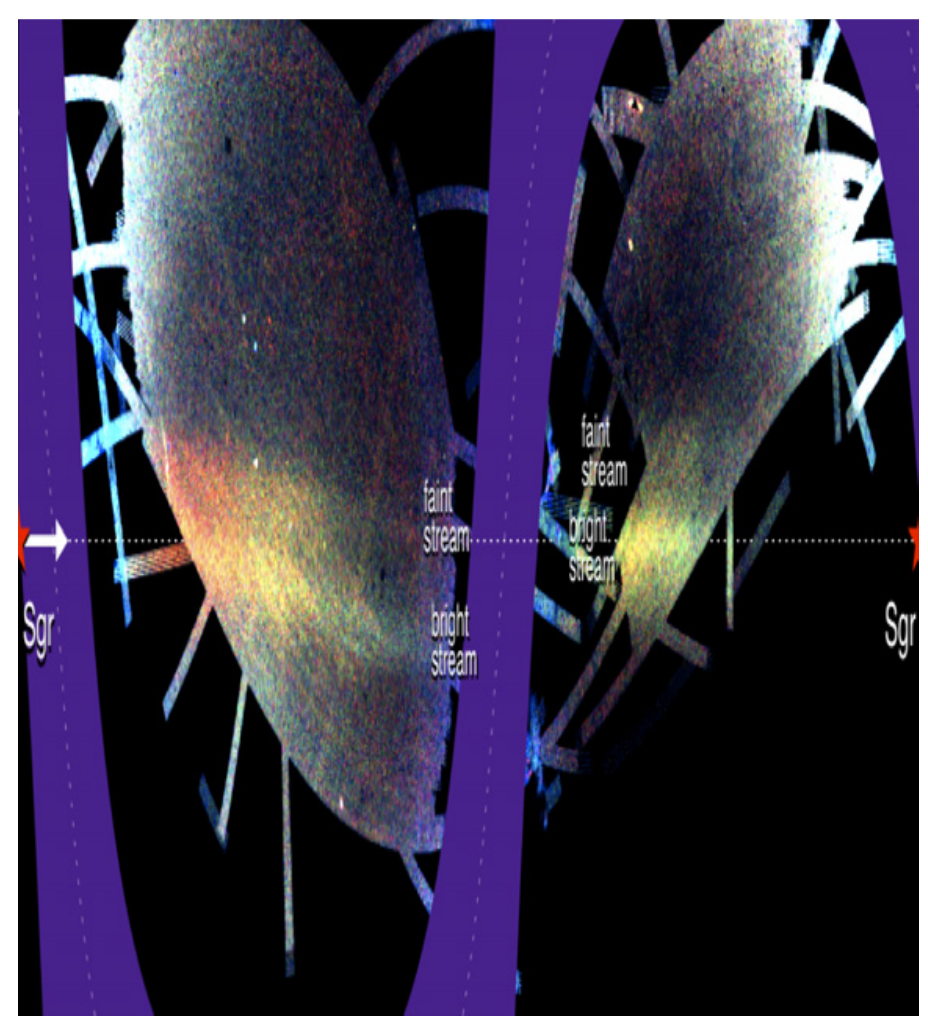

Figure 1. The Field of Streams, updated from [1]. This illustrates the extreme complexity of the outer Galactic halo, where on-going accretion is apparent.

the number count stop diverging? Lunar mass? Solar mass? $10^{9} M_{\odot}$ ? If that scale is low mass then the extreme numbers of low-mass potential wells should be the places where baryons can cool efficiently, and form stars. That is, galaxy formation should be a process of star formation in many low-mass systems, which are then merged and collected into larger systems. A hierarchy. The low mass dSph galaxies would then represent rare survivors of those very many pre-galactic systems.

This model is manifestly an extreme extrapolation. The simple existence of the galaxy massmetallicity relation tells us that when most stars form, they form in a potential well which is closely related to the potential well they occupy today. Thus baryonic gas physics must dominate. In spite of this, detailed study of the dSph, and the stellar populations in the Galaxy, is the only way to test the basic tenet of the CDM extrapolation, and find what scale, if any corresponds to that on which most early stars form.

\section{STELLAR POPULATIONS IN THE GALACTIC HALO: COMPLEXITY}

The outer Galactic halo provides clear evidence that on-going accretion is an important process in Galaxy building. The famous "Field of Streams" image derived from the SDSS imaging survey ([1]), shown here in figure 1, makes this apparent. The dominant structure is tidal tails from the Sgr dwarf galaxy ([6]). However, to illustrate the complexity consider the area where the Orphan Stream ([3]) crosses the Sgr tails. This hosts Segue 1, the most extreme ultrafaint dSph system known ([2],[10], [12]), which has the same radial velocity, internal velocity dispersion, and approximate distance as the Orphan Stream, though apparently being offset from it. It additionally is crossed by a " $300 \mathrm{~km} / \mathrm{s}$ stream", 

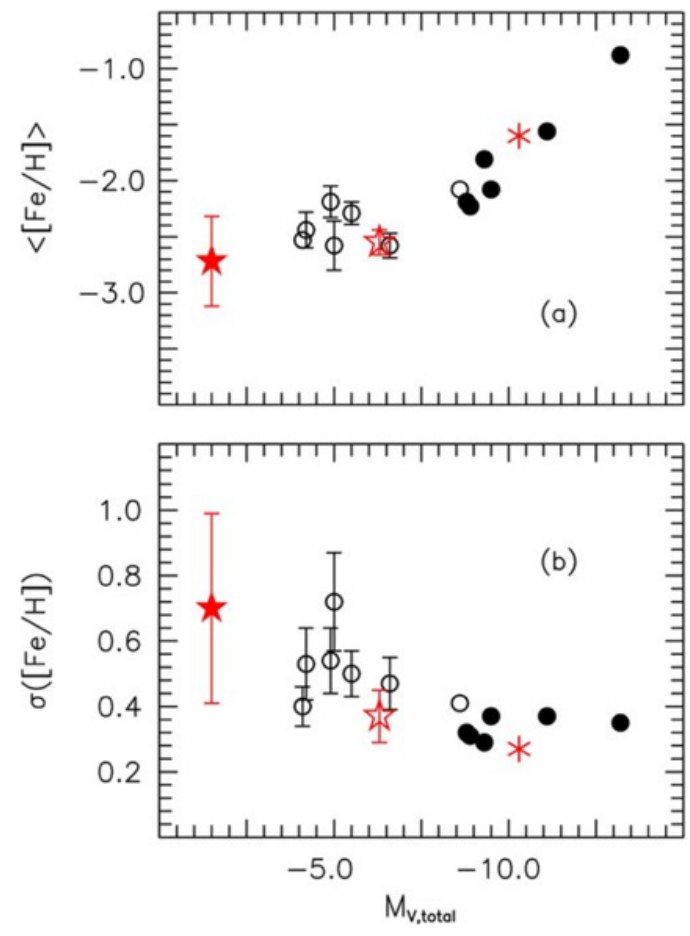

Figure 2. (a) Mean metallicity, and (b) metallicity dispersion, $\sigma[\mathrm{Fe} / \mathrm{H}]$, vs. system absolute magnitude, $\mathrm{M}_{V, \text { total }}$, for the dwarf galaxy satellites of the Milky Way and the massive globular cluster $\omega$ Cen. Figure from [10].

again of similar distance. Thus at least four structures are visible in projection on the one line of sight, with some, complex, phase-space connection between some of them.

It is then easy to suggest, as many authors do, that the entire line of sight is made up from recent accretion. In this picture the Galaxy was small until recently, and is growing still. It is also growing younger - the stars in the Sgr tail are much younger than are inner halo stars - and more metal rich - Sgr has very much higher abundance than classical Population II. However, we are here describing the very outer Galaxy. Is that representative of the rest of the Galaxy, where most stars are. The distance of Sgr, some 20 or more kpc, is perhaps 5-10 halo scale lengths from the galactic centre. Only a few percent of the halo density is located there. This is of course why we can detect such extremely faint systems as those mentioned above. But it is also a reminder that we cannot extrapolate from such systems to deductions about the majority of the Galaxy.

\section{PROPERTIES OF THE DSPH GALAXIES}

The dSph galaxies really are galaxies, with a history of extended star formation, chemical selfenrichment, and survival of a dominant dark matter halo. They extend the properties of larger galaxies smoothly to lower luminosities (eg $[5,10]$ ) as shown in fig 2 here. Figure 2 shows the both the mean and the dispersion in iron abundance of dSph member stars as a function of system luminosity. There is a very clear trend, which contains much information.

The dispersion in metallicity is always significant. This is strong evidence for internal selfenrichment in these very faint galaxies. It is both consistent with, and requires, that they be dominated by a dark matter halo. There is not enough baryonic mass, by many orders of magnitude, to retain supernova ejecta and allow self-enrichment. It imples that several to many generations of star formation took place, 


\section{EPJ Web of Conferences}

with chemical enrichment, showing these were indeed surviving star forming galaxies at very early times. The increase in dispersion with decreasing luminosity can be explained in many ways. There is a further important constraint, which allows us to limit the range of possible explanations: the observed very low scatter in element ratios in these same stars. This low scatter argues against rapid destructive star formation, and in favour of homogeneous but stochastic enrichment. Small element ratio scatter requires that each newly-forming star is made from the chemical products of a mass-averaged stellar IMF. This requires both time, to allow the lower-mass supernova progenitors to evolve and explode, and efficient mixing of the ejecta into the interstellar medium. Thus a very low star formation rate is prefered. This also allows enrichment without overly-rapid gas loss through supernova heating. Importantly, in terms of the jargon of galaxy formation models, it implies low feedback. In fact, feedback sufficiently low that the present-day dark matter halos are essentially unaffected. Thus, these CDM halos are the primordial ones, whose properties will allow us to constrain the nature of the CDM particles, as noted above.

The trend in mean metallicity with system luminosity is also interesting, and full of information. The trend establishes that the present-day system luminosity is correlated tightly with system properties when the stars were forming, and the self-enrichment was being established. That was at very early times, as these dSph all host very old stars. That is, these $\mathrm{dSph}$ galaxies retain their original luminosity: they have not been significantly tidally stripped by passage through the Milky Way potential. This is very remarkable indeed, and quite unexpected in standard CDM models. Most observable halos in those hierarchical models are seen close to disruption, after considerable tidal damage. We have here another indication of how to improve Galaxy formation, and growth of structure, models. And, of course, if the $\mathrm{dSph}$ are essentially primordial, they have not contributed many stars to the Milky Way-yet.

The other very relevant factor to consider here is of course element ratios. Many authors - for a review see, for example, [8] - have shown that stars in $\mathrm{dSph}$ galaxies have chemical element ratios which lie systematically below those of stars in the local Galactic halo, for abundances $[\mathrm{Fe} / \mathrm{H}] \geq-2$. This is well understood as a consequence of very much lower rates of star formation in the surviving $\mathrm{dSph}$ than those which happened in whatever star forming regions led to formation of the Galactic field stars (eg [4]). Again, we see that surviving dSph are not useful examples of whatever formed the Galaxy.

\subsection{The stars in dSph galaxies}

The standard explanation of the metallicity-luminosity trend follows from simple chemical evolution models. These models have one critical ingredient - the stellar IMF. The high-mass IMF determines the number of supernovae. Supernova explosion energetics are important since they provide the energy which drives mass-loss from the evolving $\mathrm{dSph}$ galaxy through galactic winds. These winds do work against the gravity of the galactic dark matter potential. Deeper potential wells imply greater energetic input to the ISM, to allow it to escape, which further implies more supernovae. More supernovae implies in turn more chemical enrichment. Hence supernova-driven winds generate a correlation between metallicity and galaxy dark matter potential, which becomes reflected in the baryonic mean abundance and number of stars formed, or system luminosity and metallicity.

The stellar IMF at low masses in dSph galaxies can be determined by directly counting stars, and compared with the IMF at comparable low masses in the Galaxy, and in globular clusters. This experiment was carried out by [14]. Their conclusion (fig 3) is that the low-mass IMF is universal, and unaffected by the environmental density over the full range of densities known in the local Universe.

The IMF at high masses cannot be derived by counting stars. It can however be derived by considering elemental abundance yields. Supernova yields are a strong function of the main-sequence mass of the progenitor. Higher-mass progenitors produce relatively more alpha elements. Thus an IMF biassed to high mass stars will generate enrichment with high $[\alpha / \mathrm{Fe}]$ values in the next generation of low-mass stars to form. This effect has been quantified, and used with observations of the $[\alpha / \mathrm{Fe}]$ values measured in metal-poor stars to determine possible changes in the high-redshift metal-poor IMF ([9]). 




Figure 3. The low-mass stellar Initial Mass Function in the UMi dSph galaxy, from [14]. The data for UMi (histogram) are compared to data for the similar metallicity globular cluster M92, and are apparently similar. This illustrates that the low-mass stellar IMF is unaffected by system density across essentially the full range available in the local Universe.

Remarkably, no difference is identifiable between the high-redshift IMF and that observed today. This is a quantification of the dramatically plateau-like relation between element ratios and $[\mathrm{Fe} / \mathrm{H}]$ across the range of abundances relevant to Galactic halo stars.

For clarity we note that the systematically low ratios of $[\alpha / \mathrm{Fe}]$ seen in dSph stars, compared to Galactic stars of comparable [Fe/H], do not indicate a varying IMF. Rather, as shown by [4], these low ratios indicate extended low-rate star formation and enrichment.

We thus can conclude that the stellar populations in dSph galaxies have an IMF which is indistinguishable from that seen locally in the Milky Way. A large hypothetical population of former dSph galaxies has not been missed since they lacked low-mass stars.

We now return to the luminosity-metallicity relation, knowing that the stellar IMF is universal. The "simple model" of galactic evolution provides the valuable concept of the "yield" and the "effective yield" of a system in which all the gas is consumed or lost. If all gas is consumed through star formation, the resulting typical metal abundance will be the yield, which is close to solar abundance. In a system with no remaining gas, but with mean abundance below solar, the mean abundance reflects an effective yield, or fraction of gas lost to winds during star formation. [All this requires a constant IMF, of course.] Thus the range of metallicities seen in $\mathrm{dSph}$ galaxies can be understood as reflecting the fraction of gas mass lost during system evolution, which reaches up to $90+\%$ for the lowest luminosity systems. This low efficiency of forming and retaining stars is reassuringly consistent with the low system luminosity, and again reflects the range of dark matter halo masses. A similarly high gas loss is required by the low metallicity of the Galactic halo.

Where did the lost gas go? Figure 4 reviews one view of the connections between the several Galactic populations. We note this is just one view, since current evidence suggests the Galactic bulge is not a "classical" bulge, but is a pseudo-bulge, arising from inner disk dynamical instability ([11]). This figure is derived from the analysis by [7]. Whatever the reality, this makes it clear that gas lost from the halo, and ant halo substructure, such as $\mathrm{dSph}$ galaxies, is unrelated to the pre-enrichment of the disks. That lost gas presumably fell into the central regions, inner disk plus whatever else lives there. The "g-dwarf" pre-enrichment problem for the bulk of the Galaxy's stars remains an unsolved problem. 


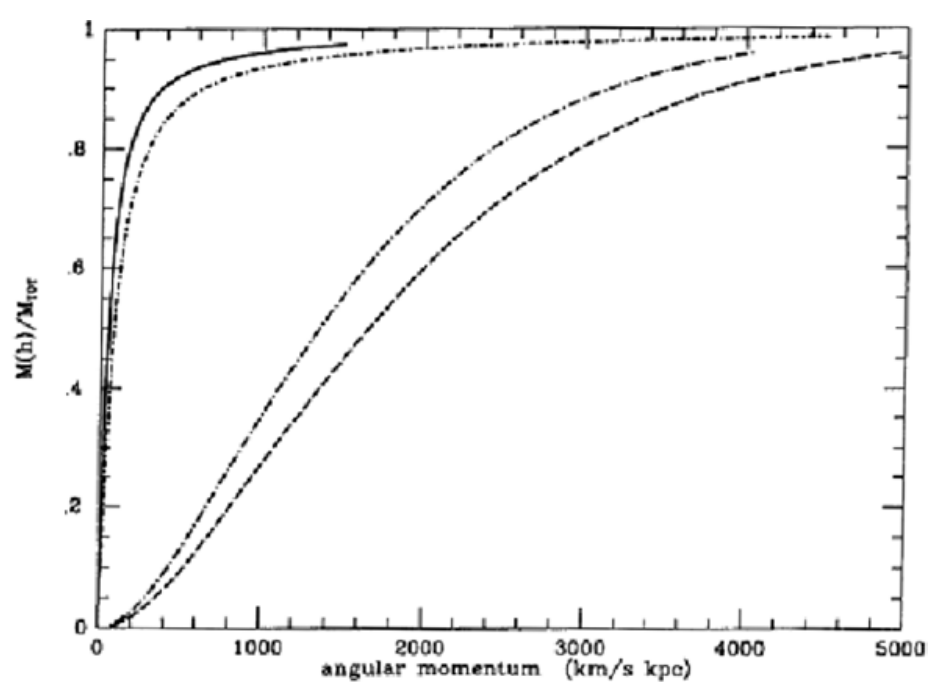

Figure 4. Normalized angular momentum distributions for a bulge-like system (solid curve), for the spheroid (short dash-dot), the thick disk (long dash-dot) and the thin disk (long dash), from [13].

\section{CONCLUSION}

It is perhaps helpful to note that we observe halos forming today in two quite different ways. One way is assembly through mergers, what one might term the Toomre method. These mergers may be very gentle, with large mass ratios, as we see in the Milky Way and in M31, or they may be near equal mass ratio mergers, which are much more violent, and lead to many famous HST images. That process involves the merger of CDM halos containing stars. The second mode of star formation is in situ, independent of any (apparent) influence by CDM sub-halos. This process is essentially one of cooling flows, sometimes perhaps cold flows. It is manifest nearby in the galaxy NGC1275, where star formation in the extended halo takes place now in cooling filaments.

The very well-established small scatter in element ratios for field halo stars over the extremely broad range of $-2.5 \leq[\mathrm{Fe} / \mathrm{H}] \leq-1$ requires that they formed from a well-mixed interstellar medium, such that essentially every star is enriched by a mass-averaged IMF supernova yield. The flatness of the relation shows that this formation process was completed in less than $\sim 1 \mathrm{Gyr}$ ([4]). It also needs to be emphasised that the Galactic halo stars have a metallicity distribution function which is, to first order, Gaussian centred near $-1.5 \mathrm{dex}$, with dispersion approximately $0.5 \mathrm{dex}$. That is, essentially the entire galactic halo is found in the metallicity range $-2 \leq[\mathrm{Fe} / \mathrm{H}] \leq-1$. The "mean" halo star is also found interior to the Solar Galactocentric radius, not in the outer halo.

Detailed studies of metal-poor stars, which now extend down to $-5 \mathrm{dex}$, are of considerable intrinsic interest, in probing the earliest stages of chemical enrichment in the Universe. Information derived from these extreme metallicity stars however tells us essentially nothing about the relevance, or otherwise, of CDM sub-halos during the formation of the mean field halo star. Very metal-poor stars tell us about formation and chemical enrichment of very metal-poor stars, not about the Galaxy.

The available evidence from chemical abundances confirms that the majority of stars in the Galactic halo formed from a well mixed system, rather early, and rather quickly. Cooling filamentary flows are a more natural way in which to meet these requirements than are very large numbers of individually small and independent systems, later disrupted. 
Assembling the Puzzle of the Milky Way

\section{References}

[1] Belokurov, V, Zucker, D., Evans, N.W., Gilmore, G. et al , ApJ 6422006137

[2] Belokurov, V, et al ApJ 6542007897

[3] Belokurov, V, et al ApJ 6582007337

[4] Gilmore, G., Wyse, R.F.G., ApJL 3671991 L55

[5] Gilmore, G., et al ApJ 6632007948

[6] Ibata, R., Gilmore, G., Irwin. M, Nature 3701994194

[7] Ibata, R., Gilmore, G., MNRAS 2751995605

[8] Koch, A. AN 3302009675

[9] Nissen, P.E., et al A\&A 2851994440

[10] Norris, J.E., Wyse, R.F.G., Gilmore, G., et al ApJ 72320101632

[11] Shen, J., Rich, R.M., Kormendy, J., et al ApJ 7202010 L72

[12] Simon, S., Geha, M., et al ApJ 733201146

[13] Wyse, R.F.G., Gilmore, G. AJ 1041992144

[14] Wyse, R.F.G., Gilmore, G., et al New Astr. 72002395 